\title{
Inducible ablation of adipocytes in adult transgenic mice expressing the $E$. coli nitroreductase gene
}

\author{
R Felmer, W Cui and A J Clark \\ Department of Gene Expression and Development, Roslin Institute, Roslin, Midlothian EH25 9PS, Scotland, UK \\ (Requests for offprints should be addressed to A J Clark; Email: john.clark@bbsrc.ac.uk) \\ (R Felmer is now at National Institute for Agricultural Research (INIA), CRI-Carillanca, PO Box 58-D, Temuco, Chile)
}

\begin{abstract}
We describe the use of an enzyme prodrug system based on E. coli nitroreductase (NTR) to achieve the specific ablation of adipose tissue. Transgenic mice expressing the NTR gene specifically in the adipose tissue were generated using the adipocyte specific promoter aP2. After treatment with the prodrug CB1954 these mice showed extensive cell depletion in all fat depots; this was directly correlated to both the dose of prodrug and the levels of NTR expression. Higher doses of CB1954 resulted in complete disappearance of visible adipose stores in some transgenic mice. These mice exhibited an impaired ability to thermoregulate body temperature. Lower doses of CB1954 resulted in a partial reduction of the adipose tissue
\end{abstract}

leaving non-expressing cells that escape ablation. These animals show normal levels of blood glucose and triglycerides but have reduced leptin levels. After 30 days they were able to regenerate the fat depots and leptin levels returned to normal but, interestingly, no NTRexpressing cells were detectable. The present model provides a new approach to manipulate the number of adipocytes at different stages of mouse development and provides a new system for the study of fat metabolism especially in abnormal conditions such as obesity and its modulation through manipulation of the target cell population.

Journal of Endocrinology (2002) 175, 487-498

\section{Introduction}

Adipocytes play a central role in maintaining the energy balance of higher organisms and fat depots are a major endocrine tissue that regulates body mass. These cells have the ability to store energy in the form of triglycerides, and can mobilise this energy reserve upon demand, to provide the needed physiological fuel for other cell types. Each of these specific lipogenic and lipolytic functions are carried out by two types of adipose tissue known as white adipose tissue (WAT) and brown adipose tissue (BAT). In contrast to the development of BAT, which takes place mainly before birth (Housteck et al. 1988), WAT first appears at birth (Ailaud and Hauner 1998) and continues growing throughout life. BAT is distinguished from WAT by the unique presence of the uncoupling protein (UCP), a mitochondrial protein transporter that uncouples oxidative metabolism from ATP synthesis. This capacity permits BAT to expend calories unrelated to the performance of work, the net result being the generation of heat (Himms-Hagen 1990).

The discovery of regulatory elements (Ross et al. 1990) that can direct tissue specific expression to adipocytes opened up the possibility of experiments regulating the fatness of laboratory and domestic animals. This has led to the study of the metabolism and physiology of this tissue in different transgenic models. Genetic ablation is a powerful tool that allows the removal of specific cell types by targeting the expression of intracellular toxins (Breitman et al. 1987). In this approach, expression of a toxic gene or, secondly, a gene whose product can convert an otherwise harmless prodrug into cytotoxic species (Borrelli et al. 1989), are targeted to specific cell types by using the appropriate regulatory sequences. In the former case, ablation of the targeted cells is achieved as soon as the gene switches on, while in the latter approach ablation is induced only after downstream administration of the prodrug, thus allowing investigators to control the timing of ablation.

Genetic ablation approaches using diphtheria toxin have already been used to generate a number of transgenic mouse models with decreased white and brown adipose tissue (Ross et al. 1993, Lowell et al. 1993, Burant et al. 1997). One problem associated with this system derives from its extreme cytotoxicity. Although this ensures that cells expressing low levels will be killed, it also means that any leakiness from the transcriptional regulatory elements resulting in ectopic expression of the toxin could lead to embryo lethality or deleterious effects that compromise the analysis of the resulting transgenic animals. Secondly, this approach does not allow the investigator control over the timing of cell ablation and so it is not feasible to assess the 
consequences of ablation at defined developmental timepoints. A solution to this problem is to use a gene prodrug system such as HSV1tk/ganciclovir so that ablation is effected only when the prodrug is administered (Borrelli et al. 1989). Ablation using HSV1 tk/ganciclovir, however, normally requires dividing cells and, therefore, is not suitable for tissues that are primarily post-mitotic, such as mature fat depots. We, therefore, assessed the use of an alternative system for cell ablation that overcomes some of these limitations. This is based on the use of the E. coli nitroreductase (NTR) enzyme which activates certain nitro-compounds, such as the antitumour drug CB1954, into potent cytotoxic agents capable of DNA cross-linking (Knox et al. 1993). The system is effective in both dividing and non-dividing cells (Clark et al. 1997, Isles et al. 2001) and cell killing is inducible. It provides the means to control the timing and duration of the toxic insult and to assess the potential for regeneration of an ablated cell population after a prodrug free period.

To study the consequences of conditionally ablating adipocytes we generated transgenic mice expressing the E. coli NTR gene in the adipose tissue using the adipocyte specific promoter aP2 (Ross et al. 1990). These mice showed specific expression of NTR in adipocytes although the pattern of transgene expression was variegated. Upon CB1954 treatment, there was a dose-dependent depletion of adipocytes in all major adipose depots whereas other tissues remained normal or comparable to control mice. At high doses this disrupted the ability of the animals to thermoregulate and led to their morbidity. At lower doses the ablation of adipocytes was incomplete and had little effect on the circulating levels of glucose or triglycerides. Leptin levels were, however, reduced, and a small increase in free fatty acids was also observed. Four weeks post-ablation the fat pads had regenerated but, interestingly, did not now comprise any NTR expressing cells.

\section{Materials and Methods}

\section{Plasmid construction}

The plasmid directing the fat-specific expression of E. coli NTR gene was constructed as follows: A $1.55 \mathrm{~kb}$ fragment containing the E. coli NTR gene and the poly A (pA) signal from SV40 was liberated from the plasmid pWR6, kindly provided by Dr W. Cui (Clark et al. 1997), by double digestion with Hind III/Xba I and end filled ligated into the unique Sma I site of the aP2 promoter/enhancer plasmid (kindly provided by Dr Reed Graves) (Ross et al. 1990) to generate the aP2-NTR plasmid. For microinjection a $6.95 \mathrm{~kb}$ fragment containing the $\mathrm{aP} 2$ promoter, NTR and pA (SV40) was obtained free of any vector sequences by double digestion Sal I/Not I and gel purification.
Mice

Transgenic mice were produced by microinjection of the linearised $6.95 \mathrm{~kb}$ fragment into male pronuclei from the $\mathrm{C} 57 \mathrm{BL} / 6 \mathrm{j} \times \mathrm{CBA}$ strain of mice. Transgenic offspring were screened by PCR on tail DNA with transgenespecific primers 5'-GCAGATCAAAACGCTACTGCA ATAC3'/5'AGCCTTTCTCTTTCAGACCAAATTC-3'. Mice were housed in colony cages and maintained on a $12 \mathrm{~h}$ light $/ 12 \mathrm{~h}$ dark cycle at $20^{\circ} \mathrm{C}$ and were fed ad libitum. All experimental procedures involving mice were carried out according to Home Office guidelines (Animals (Scientific Procedures) Act 1986).

\section{Prodrug administration}

CB1954 was suspended in arachis oil containing 10\% acetone at concentrations of 2 and $4 \mathrm{mg} / \mathrm{ml}$. Age- and sex-matched transgenic and non-transgenic mice (7 weeks old), were weighed and injected intraperitoneally.

\section{Body and organ weight}

To study the effects of CB1954 administration on body weight during administration, mice were weighed daily until the end of the experiment. For organ weight measurements different white fat pads (epididymal/ parametrial, retroperitoneal, inguinal), brown fat (interscapular region), heart, liver, spleen and kidney were dissected and weighed.

\section{RNA preparation and Northern blot analysis}

Tissues were removed and frozen in liquid nitrogen after mice were killed. RNA was extracted with RNAzol B (Biogenesis, Poole, UK) following the manufacturer's instructions. Fifteen micrograms of total RNA were fractioned on $1.5 \%$ formaldehyde/agarose gels and blotted onto nylon membranes (Boehringer Manheim). The blots were hybridised at $65^{\circ} \mathrm{C}$ overnight in $0.5 \mathrm{M}$ sodium phosphate, $7 \%$ SDS with ${ }^{32} \mathrm{P}$-labelled cDNA probes and washed in $2 \times$ SSC, $0 \cdot 1 \%$ SDS for $10 \mathrm{~min}$, followed by one wash of $15 \mathrm{~min}$ in $0.2 \times \mathrm{SSC}, 0 \cdot 1 \% \mathrm{SDS}$. The membranes were visualised using Phosphorimager (Molecular Dynamics) and AGFA x-ray film.

\section{Western blot analysis}

Tissues were homogenised in lysis buffer $(25 \mathrm{mM}$ Tris $\mathrm{pH}$ 7·4, $150 \mathrm{mM} \mathrm{NaCl}, 1 \% \mathrm{NP}-40,1 \mathrm{mM}$ EDTA, $1 \mathrm{mM}$ EGTA, $1 \mathrm{mM}$ dithiothreitol (DTT) and $9 \mu \mathrm{g} / \mathrm{ml}$ phenylmethylsulphonyl fluoride (PMSF)), and proteins were extracted by centrifugation for $5 \mathrm{~min}$ at $4{ }^{\circ} \mathrm{C}$. Proteins were transferred to nitrocellulose after electrophoresis by one-dimensional SDS-PAGE. To block non-specific proteins, the membrane was incubated in a solution of Tris 


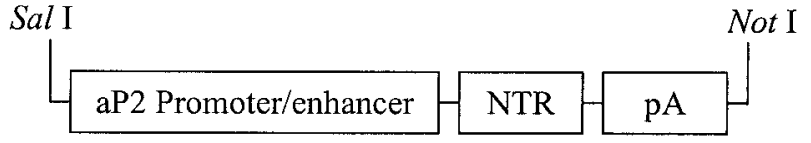

\section{aP2-NTR plasmid $6.95 \mathrm{~kb}$}

Figure 1 Transgene construct. The adipocyte specific aP2 promoter enhancer (Ross et al. 1990) was used to drive the expression of the E.coli nitroreductase gene to the adipose tissue of transgenic mice. For microinjection, a $6.95 \mathrm{~kb}$ fragment comprising the aP2 promoter, the NTR coding sequence and the SV40 poly A site was obtained free of any vector sequences by double digestion Sal I/Not I and gel purified before microinjection into pronuclear stage eggs from superovulated $(\mathrm{C} 57 \mathrm{Bl} / 6 \mathrm{j} \times \mathrm{CBA}$ F1) females. buffer saline/Tween (TBS-T) containing 3\% BSA for $1 \mathrm{~h}$ at room temperature. The nitrocellulose membrane was exposed to a polyclonal anti-NTR antibody diluted in 1:1500 in blocking solution containing $1 \%$ BSA for $1.5 \mathrm{~h}$ at room temperature. The membrane was then washed five times with TBS-T at 5 min intervals. The primary antibody was visualised using peroxidase-conjugated horseradish peroxidase (HRP)-anti-rabbit IgG secondary antibody (1:5000 dilution in TBS-T/1\% BSA) for $1 \mathrm{~h}$ at room temperature and enhanced chemiluminescence (ECL) detection reagents (Amersham).

\section{Histology}

White adipose tissue (epididymal/parametrial), interscapular brown fat and skin were removed and fixed in

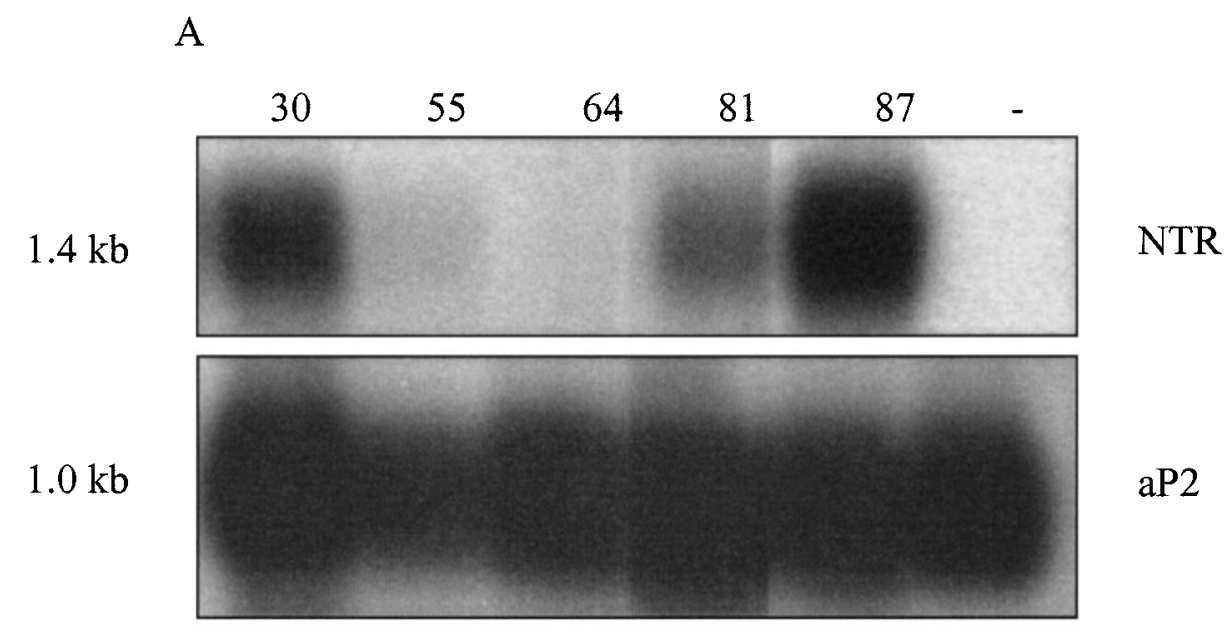

B

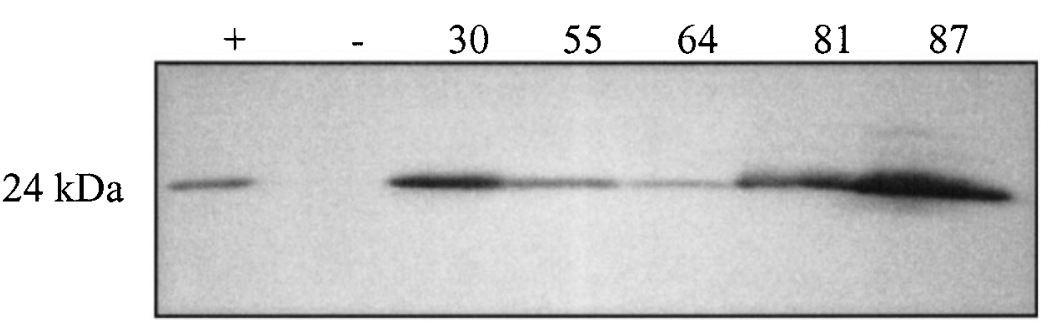

Figure 2 Transgene expression in different lines of mice expressing E.coli NTR. (A) Northern blot of $10 \mu \mathrm{g}$ total RNA extracted from WAT of five different transgenic lines (lines 30, 55, 64, 81 and 87 respectively). The blot was hybridised with a probe to detect NTR in the upper panel and then stripped and re-hybridised with an adipocyte specific probe (aP2) as loading control (lower panel). (B) Western blot analysis. Total protein tissue extracts $(20 \mu \mathrm{g})$ were prepared from adipose tissue of one member of each of the transgenic lines that showed expression by Northern blot (above). The blot was probed with a polyclonal antibody directed to the E. coli NTR. +, positive control (mammary gland RNA from a previously described mouse line expressing NTR in this tissue (Clark et al. 1997)); - , non-transgenic control. 
A
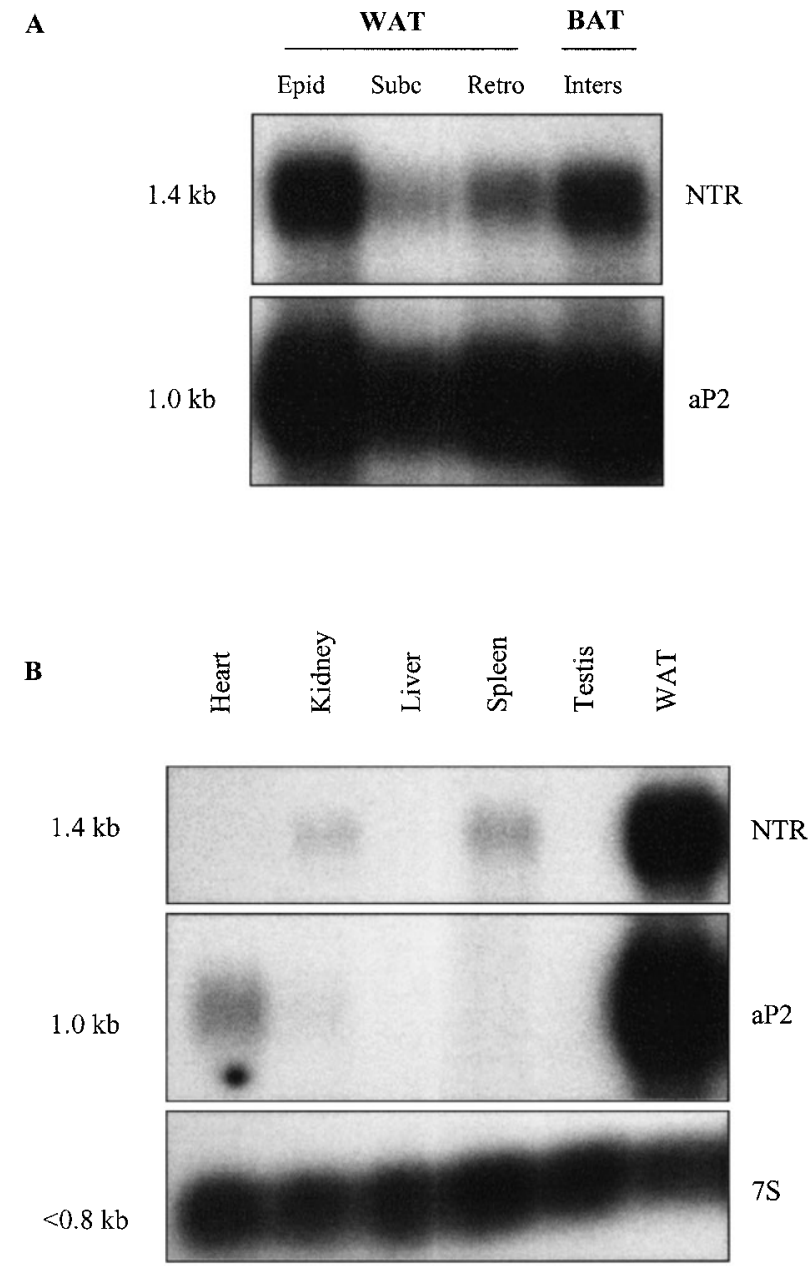

Figure 3 Expression analysis of NTR mRNA in different tissues. (A) Analysis of the expression of NTR in different adipose depots. Total RNA was collected from different fat depots; Epid, epidydimal; Subc, subcutaneous; Retro, retroperitoneal white fat (WAT) and Inters, interscapular brown fat (BAT). The blot was hybridised with an NTR probe in the upper panel and then stripped and re-hybridised with an adipocyte specific probe (aP2) as loading control. (B) Analysis of the ectopic expression of NTR in different tissues. Total RNA was collected from different tissues of a transgenic mouse (line 87) and the blot hybridised with an NTR probe (upper panel) and then stripped and re-hybridised with the adipocyte specific probe aP2 (middle panel) as control for contaminating fat, stripped again and re-hybridised with a $7 S$ probe (lower panel) as loading control.

neutral buffered formalin (NBF) for at least $24 \mathrm{~h}$ before embedding in paraffin using standard procedures. Five to eight micrometre sections were cut and stained with Mayer's haematoxylin-eosin staining and mounted in DPX mounting medium.

\section{Immunohistochemistry}

Dewaxed sections of adipose tissue were pretreated with $0 \cdot 1 \%(\mathrm{w} / \mathrm{v})$ trypsin in PBS for $10 \mathrm{~min}$ at $37^{\circ} \mathrm{C}$ for antigen retrieval. Endogenous peroxidase was blocked with 1\% $(\mathrm{v} / \mathrm{v})$ hydrogen peroxide. After blocking non-specific antibody binding in 5\% (v/v) normal swine serum (Dako, Glostrup, Denmark) the sections were incubated for $1.5 \mathrm{~h}$ at $37^{\circ} \mathrm{C}$ with the primary antibody (anti-NTR) diluted in blocking solution (1:300). After washing in PBS, sections were incubated with the secondary antibody for $1 \mathrm{~h}$ at room temperature. (1:200 swine anti-rabbit Ig biotinylated antibody Dako; E0353). Sections were washed in PBS and incubated with streptavidin-peroxidase conjugate (Zymed, San Francisco, CA, USA) at 1:2000 for $30 \mathrm{~min}$ at room temperature in the dark. The colour was developed by incubation with a diaminobenzidine (DAB) $/ \mathrm{H}_{2} \mathrm{O}_{2}$ solution for $10 \mathrm{~min}$. Sections were counterstained with haematoxylin, dehydrated and mounted in DPX and visualised by light microscopy.

\section{Blood analysis}

Animals were killed by cervical dislocation between 1500 and $1800 \mathrm{~h}$, and trunk blood was collected. Plasma leptin levels were determined by using radioimmunoassay kits with rat insulin and recombinant leptin respectively, as standards (Linco Research Immunoassay, St Charles, MO, USA). Plasma triglyceride levels were assayed with commercially available kits (Sigma and Boehringer Mannheim). Whole blood glucose level was measured by using a One-Touch Profile glucose meter (Lifescan, Milipitas, CA, USA).

\section{Statistical analysis}

One-way ANOVA and Student's $t$-test were use for statistical evaluation using the MINITAB program, with $P<0.05$ considered as significant. All results were expressed as means \pm S.E.M. unless indicated otherwise in the Figures.

\section{Results}

aP2 promoter targets NTR expression to adipose tissue in transgenic mice

Expression of NTR gene was driven by a $5.4 \mathrm{~kb}$ fragment containing the adipose-specific promoter/enhancer of the gene encoding aP2 (Ross et al. 1990). Microinjection of the transgene (Fig. 1) resulted in twelve transgenic founders from 97 live births. All lines had approximately normal litter sizes (i.e. 8-12 pups per litter) and no obvious phenotype was observed as a result of the transgene integration. Eleven founders transmitted the transgene into the offspring and five of these had detectable levels of expression (Fig. 2A). Further analysis of tissue samples by SDS-PAGE followed by Western blotting with a polyclonal antibody to NTR revealed a single band of 
A

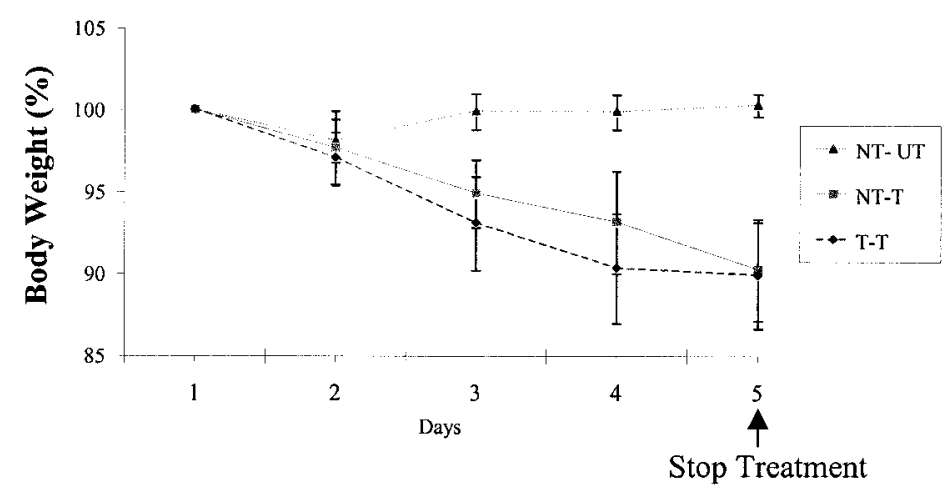

B

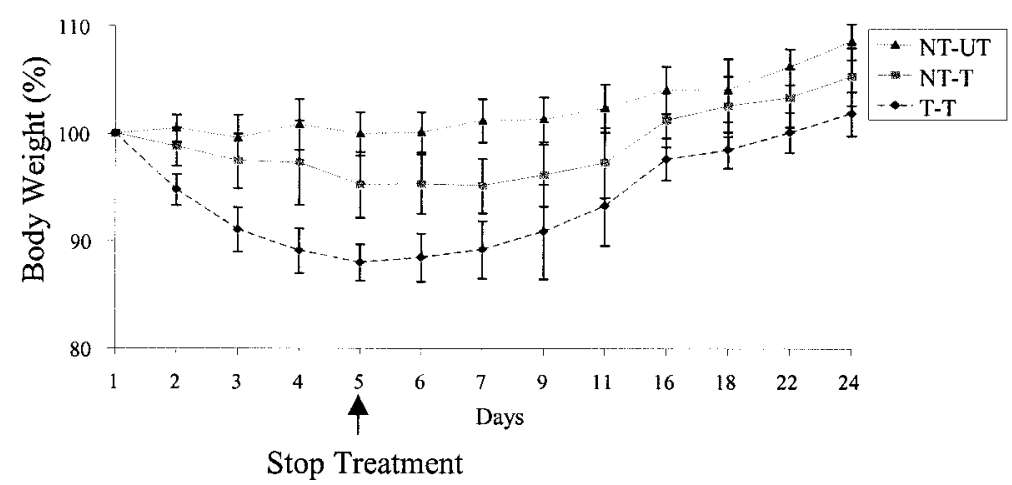

Figure 4 Measurement of body weight during and after treatment with CB1954. Groups of transgenic and non-transgenic male mice at the age of 7 weeks were injected with the $5 \times 20 \mathrm{mg} / \mathrm{ml}$ prodrug and killed on day 5 (Group A) or after a 30 day recovery period (Group B). The mean \pm S.D. body weights are plotted. NT-UT: non-transgenic untreated; NT-T: non-transgenic treated; T-T: transgenic treated.

approximately $24 \mathrm{kDa}$, the predicted $M_{\mathrm{r}}$ for NTR (Fig. $2 \mathrm{~B}$ ) and the levels of NTR mRNA correlated to those of the NTR enzyme.

One line (line 87), which has approximately 25 copies of the transgene (data not shown) and the highest level of nitroreductase expression was selected for a detailed analysis. High levels of NTR mRNA were observed in a number of different fat depots comprising WAT or BAT (Fig. 3A). To investigate the possibility of ectopic expression in other cell types Northern blotting analysis was carried out on RNA samples prepared from a wide variety of tissues including heart, kidney, liver, spleen and testes (Fig. 3B). This indicated that transgene expression is normally confined to the adipose cells. In some cases, a

Table 1 Anatomical characteristics of mice after treatment with prodrug CB1954

\begin{tabular}{|c|c|c|c|c|c|c|c|c|}
\hline & $\begin{array}{l}\text { Body weight } \\
\text { grams }\end{array}$ & $\begin{array}{l}\text { Gonadal } \\
\text { (WAT) } \\
(\%)\end{array}$ & $\begin{array}{l}\text { Inguinal } \\
\text { (WAT) } \\
(\%)\end{array}$ & $\begin{array}{l}\text { Intersc. } \\
\text { (BAT) } \\
(\%)\end{array}$ & $\begin{array}{l}\text { Liver } \\
(\%)\end{array}$ & $\begin{array}{l}\text { Kidney } \\
(\%)\end{array}$ & $\begin{array}{l}\text { Spleen } \\
(\%)\end{array}$ & $\begin{array}{l}\text { Heart } \\
(\%)\end{array}$ \\
\hline $\begin{array}{l}\text { T-T } \\
(n=4)\end{array}$ & $\begin{array}{c}15 \cdot 6 \pm 0 \cdot 6 \\
*\end{array}$ & $\begin{array}{c}0 \cdot 07 \pm 0 \cdot 01 \\
\Psi\end{array}$ & $\begin{array}{c}0.08 \pm 0.01 \\
\Psi\end{array}$ & $0 \cdot 18 \pm 0 \cdot 05$ & $4 \cdot 50 \pm 0 \cdot 1$ & $0 \cdot 77 \pm 0 \cdot 03$ & $\begin{array}{c}0 \cdot 20 \pm 0 \cdot 01 \\
*\end{array}$ & $0 \cdot 52 \pm 0 \cdot 05$ \\
\hline $\begin{array}{l}\text { NT-T } \\
(n=2)\end{array}$ & $\begin{array}{c}15 \cdot 1 \pm 0 \cdot 3 \\
*\end{array}$ & $0 \cdot 17 \pm 0 \cdot 01$ & $0 \cdot 32 \pm 0 \cdot 05$ & $0 \cdot 27 \pm 0 \cdot 01$ & $4 \cdot 7 \pm 0 \cdot 7$ & $0 \cdot 70 \pm 0 \cdot 05$ & $\begin{array}{c}0 \cdot 25 \pm 0 \cdot 01 \\
*\end{array}$ & $0.56 \pm 0.02$ \\
\hline $\begin{array}{l}\text { NT-UT } \\
(n=3)\end{array}$ & $18 \cdot 0 \pm 0 \cdot 4$ & $0 \cdot 15 \pm 0 \cdot 01$ & $0 \cdot 42 \pm 0 \cdot 02$ & $0 \cdot 23 \pm 0 \cdot 04$ & $4 \cdot 60 \pm 0 \cdot 2$ & $0 \cdot 71 \pm 0 \cdot 06$ & $0 \cdot 34 \pm 0 \cdot 05$ & $0.58 \pm 0.02$ \\
\hline
\end{tabular}

Data are means \pm S.E.M. Animals were transgenic and non-transgenic littermates of 7 weeks old. The organ weights were measured as a percentage of the animal's body weight. Probabilities $(P)$ are from one-way ANOVA $\left({ }^{*}=0 \cdot 05 ; \Psi=0 \cdot 0001\right)$. Groups with significant differences are shown. T-T: transgenic mice treated with CB1954; NT-T: non-transgenic mice treated with CB1954; NT-UT: non-transgenic mice untreated. 
A
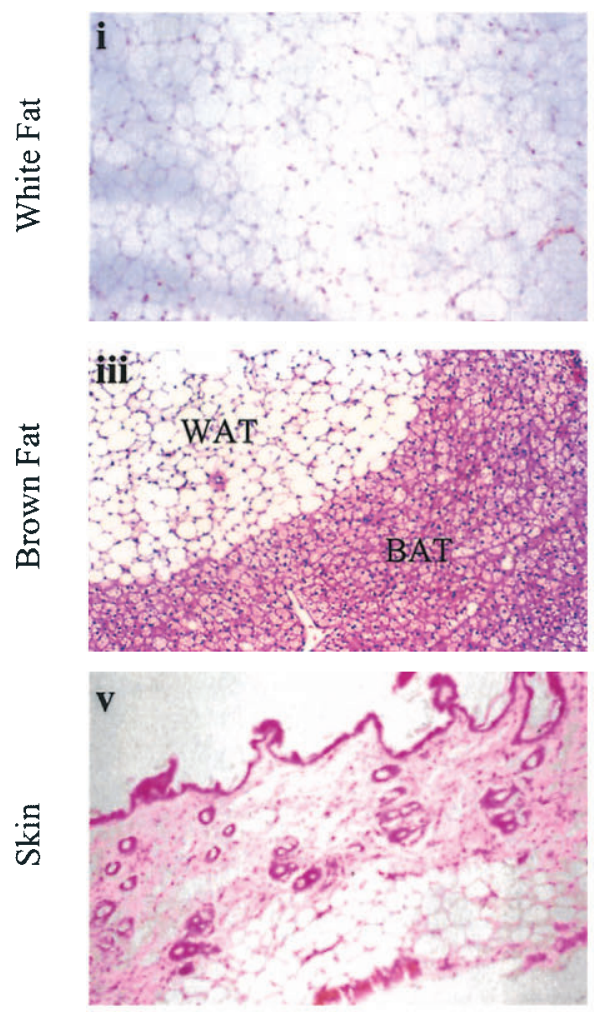

Transgenic
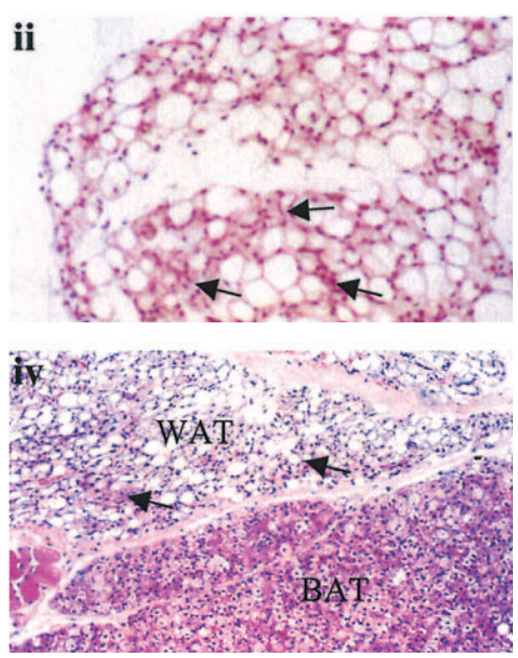

vi

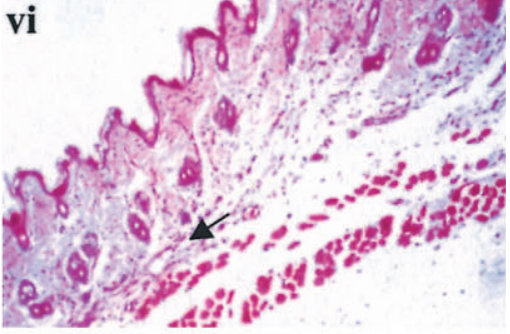

B
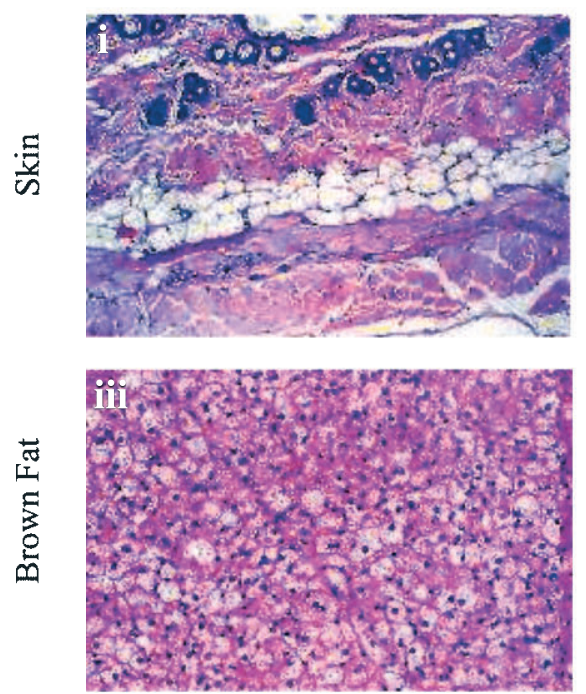

Transgenic
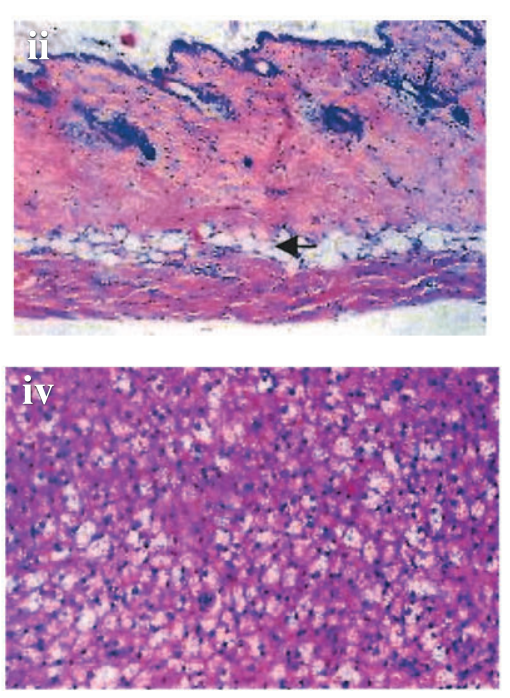
trace of NTR signal is observed in other tissues and we interpret this as corresponding to contaminating fat, since a probe to the endogenous aP2 mRNA also hybridised to RNA from these tissues (Fig. 3B, middle panel). Only the spleen showed a trace signal of NTR which was not accounted for by contaminating fat. The nature of this putative leaky expression is not clear, but may be due to effects of the transgene integration site, since the same promoter sequence has been used previously in a number of transgenic models without ectopic expression being reported (Ross et al. 1993, Lowell et al. 1993, Shimomura et al. 1998, Moitra et al. 1998).

\section{aP2/NTR transgenic mice show depletion of adipocytes after CB1954 treatment}

All mice injected with CB1954, regardless of their genotype, showed temporary weight loss (Fig. 4). Weight loss has also been observed in other experiments involving mice that have been injected with CB1954 (Drabek et al. 1997) and it has been suggested that this effect is partly due to the effects of CB1954 on NTR expressing bacteria in the gut (Clark et al. 1997). Transgenic mice receiving the higher dose of $40 \mathrm{mg} / \mathrm{kg}$ body weight showed acute signs of morbidity, including hunching suggesting that their ability to thermoregulate was compromised. Measurement of the rectal body temperature, during the 3 day course of injections, showed a drop in temperature of approximately $5{ }^{\circ} \mathrm{C}\left(32^{\circ} \mathrm{C} \pm 0.5\right)$ after 3 days, which was not observed in the control groups $\left(37^{\circ} \mathrm{C} \pm 0 \cdot 6\right)$. These animals were humanely killed as soon as signs of morbidity were evident. The hypothermic response was not observed in wild-type mice injected with the prodrug, or when transgenic mice received a lower dose $(20 \mathrm{mg} / \mathrm{kg}$ body weight $)$ of CB1954.

Autopsy of transgenic mice treated with the higher dose ( $40 \mathrm{mg} / \mathrm{kg}$ body weight) in a regimen of three injections, revealed a considerable reduction in the size of the adipose stores. Some of the transgenic mice showed complete disappearance of any visible adipose stores including parametrial (gonadal), inguinal and retroperitoneal, with other mice also showing a reduction of up to 60 and $80 \%$ wet weight of the parametrial and inguinal fat pads respectively (Table 1). Transgenic mice treated with the lower dose $(20 \mathrm{mg} / \mathrm{kg}$ body weight), also showed a reduction in the adipose tissue although this effect was not as marked as with the higher dose (data not shown).

\section{Histological changes in fat of transgenic mice treated with CB1954}

WAT is mostly formed by cells with a single large lipid droplet that occupies most of the cytoplasmic space, pushing the nucleus to the cell periphery. In contrast, BAT has a dense cytoplasm with multilocular lipid droplets, caused by its high content of mitochondria. Histological sections of the inguinal fat pads stained with haematoxylin and eosin showed mature adipocytes of uniform size each of which contained a characteristic unilocular lipid droplet in control mice (Fig. 5A, i). In contrast, remaining inguinal fat pads of transgenic mice contained a mixture of mature and immature adipocytes with some spaces in the sections where adipocytes have disappeared (Fig. 5A, ii). In contrast to wild-type WAT, BAT of control mice contained large areas of typical mitochondria rich eosinophilic cells with multiple lipid droplets as well as regions of white fat (Fig. 5A, iii). Histological examination of the brown fat in transgenic mice showed disappearance of the white fat that covers the brown fat as the mice ages but we could perceive no major effects on brown fat itself (Fig. 5A, iv). Histological sections of the skin also showed a considerable reduction of adipocytes in transgenic mice and in some instances complete disappearance of any visible adipocytes was observed (Fig. 5A, vi) without any obvious necrosis. This effect was correlated with the dose of CB1954 used and there was not such a marked reduction of adipocytes when lower doses of CB1954 were administered (Fig. 5B). Tunel assays carried out on paraffin embedded sections of adipose tissue of mice treated with the prodrug, showed in situ death of adipose cells by apoptosis (data not shown) confirming our previous observations in transgenic mice expressing NTR in the luminal cells of the mammary gland (Clark et al. 1997, Cui et al. 1999).

\section{Physiological consequences of ablation and regeneration of adipose tissue}

We studied the consequences of the adipose tissue ablation and the ability of this tissue to regenerate after a prodrug

\footnotetext{
Figure 5 Histology of different tissues after treatment with CB1954. (A) Histological sections of white fat (i and ii), interscapular brown fat (iii and iv), and skin ( $v$ and vi) from 7-week-old female transgenic (ii, iv, and vi) and non-transgenic (i, iii, and v) littermates treated with the higher dose of $40 \mathrm{mg} / \mathrm{kg}$ body weight. White adipose tissue in different tissues of transgenic mice showed reduction of adipose cells (arrows) compared with wild-type mice. Tissues were fixed in NBF and paraffin-embedded sections stained with haematoxylin and eosin. (B) CB 1954 effects are dose dependent. Histological sections of skin (i and ii) and interscapular brown fat (iii and iv) of wild-type and transgenic mice treated with a lower dose of CB1954 of $20 \mathrm{mg} / \mathrm{kg}$ body weight. Compared with the wild-type, transgenic mice showed a reduction of adipocytes in the skin but this effect was not as marked as with a higher dose of the prodrug. No major effect is observed in the brown fat of both mice. Tissues were fixed in NBF and paraffin-embedded sections stained with haematoxylin and eosin. Original magnification: $\times 100$.
} 


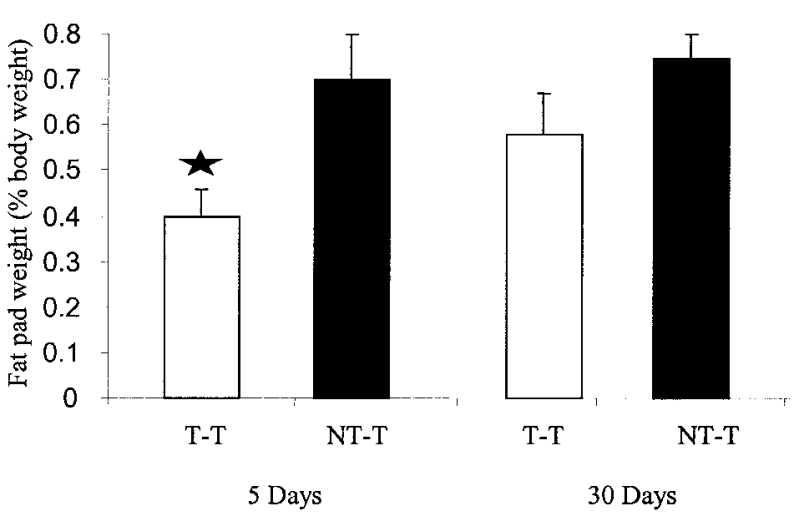

Figure 6 Analysis of the fat pad weight. Fat pad weight, as a measure of adipose tissue regeneration, was compared in transgenic (T-T, $n=8$ first group, and $n=6$ second group respectively) and non-transgenic (NT-T, $n=5$ both groups respectively) littermate mice treated ( $\mathrm{T}$ ) with $20 \mathrm{mg} / \mathrm{kg}$ body weight for 5 days and in a second group of mice treated as above but left to recover for 30 days. A Student's two-tailed unpaired $t$-test was used for statistical analysis showing significance $(P=0.02)$ in the transgenic group killed on day 5 , but not in the group killed after 30 days.

free period of 30 days. Two groups of male mice were injected with doses of $20 \mathrm{mg} / \mathrm{kg}$ body weight for 5 days and then killed on day 5 (first group) and 28 days after the last injection (second group). The lower prodrug dosage was chosen because of the morbidity associated with the $40 \mathrm{mg} / \mathrm{kg}$ dose. The extent of ablation was assessed by monitoring the change in wet weight of the epididymal fat pad as a percentage of body weight. This trait has been shown previously to be highly correlated with total body fat percentage in mice (Rogers \& Webb 1980). The results of this analysis (Fig. 6) confirmed a quantifiable effect of the lower dose of CB1954, a reduction of approximately $40 \%$ wet weight of the epididymal pad $(n=8)$ was observed when compared with the non-transgenic group $(n=5)$ also treated with the prodrug $(P=0 \cdot 02)$. After 30 days of prodrug free period, the mass of the epidymal fat pad increased from 0.40 to $0.58 \%$ in the second group of treated transgenic mice $(n=6)$ and no significant difference was observed with the controls $(n=5)$. Immunohistochemistry experiments carried out on sections of the adipose tissue of these mice showed no staining in the wild-type mice, whereas patchy expression of NTR, exclusively located within the adipocytes was observed in the transgenic mice (Fig. 7, i and 7, ii respectively). We have previously reported that this antibody is specific for NTR-expressing cells in a number of different transgenic mouse models (Clark et al. 1997, Cui et al. 2001, Isles et al. 2001). Analysis of sections of adipose tissue, after treatment of these mice for 5 days with CB1954, showed a small remaining number of NTR-expressing cells (Fig. 7, iii). Immunostaining was also observed between the cells, suggesting that they were dying and being removed by the phagocytic machinery. After the 30 days prodrug free period, no NTR staining whatsoever was observed despite some non-specific staining within the blood vessels (see arrows in Fig. 7, iv), indicating that all NTR-expressing cells had been killed and that none had been regenerated.

In order to investigate the physiological consequences of the ablation of adipose tissue in transgenic mice treated with CB1954, a number of parameters were measured in the plasma of these mice after treatment with the prodrug. These parameters included total blood glucose levels, and plasma triglyceride and leptin levels (Fig. 8). Because of the limited amount of material available from individual mice, pooled samples of plasma were used, except for glucose where it was feasible to make individual determinations. After CB1954 administration blood glucose levels are preserved and remained comparable to the controls indicating no major problems in withstanding the reduction of adipose tissue. Triglycerides, which are carried in the circulation as lipoprotein particles, were slightly elevated although this difference was not significant with any of the control groups. Leptin, which is a hormone secreted by both white and brown adipose tissue cells in proportion to fat mass (Flier 1997), was reduced in those transgenic mice treated with the prodrug thus confirming this hormone as an adiposity indicator. However, after a 30 day prodrug free period the levels of this hormone returned to normal indicating the regeneration of the ablated tissue.

\section{Discussion}

In this report, we use an inducible system to genetically manipulate the number of adipocytes in adipose tissue by targeting E. coli NTR gene expression to these cells with the adipocyte specific promoter aP2. CB1954 administration produced selective killing of adipocytes in transgenic mice by a mechanism involving apoptosis (data not shown), confirming our preliminary findings with a preadipocyte cell line (R Felmer \& A J Clark, unpublished observations) and also our previous publications (Clark et al. 1997).

The magnitude of the ablation of tissue was directly correlated to the dose regimen used and morbidity effects observed with the mice. Whereas a lower dose of $20 \mathrm{mg} / \mathrm{kg}$ body weight produced a partial reduction of adipocytes with no signs of morbidity, a higher dose of $40 \mathrm{mg} / \mathrm{kg}$ body weight in a regimen of three injections resulted in a complete disappearance of any visible adipose depots in a number of transgenic mice, although some variability could also be observed in other mice with a less marked effect of the prodrug. We attribute this variability to variegated transgene expression since immunohistochemical analysis carried out in sections of adipose tissue showed patches of NTR expressing cells alongside non- 

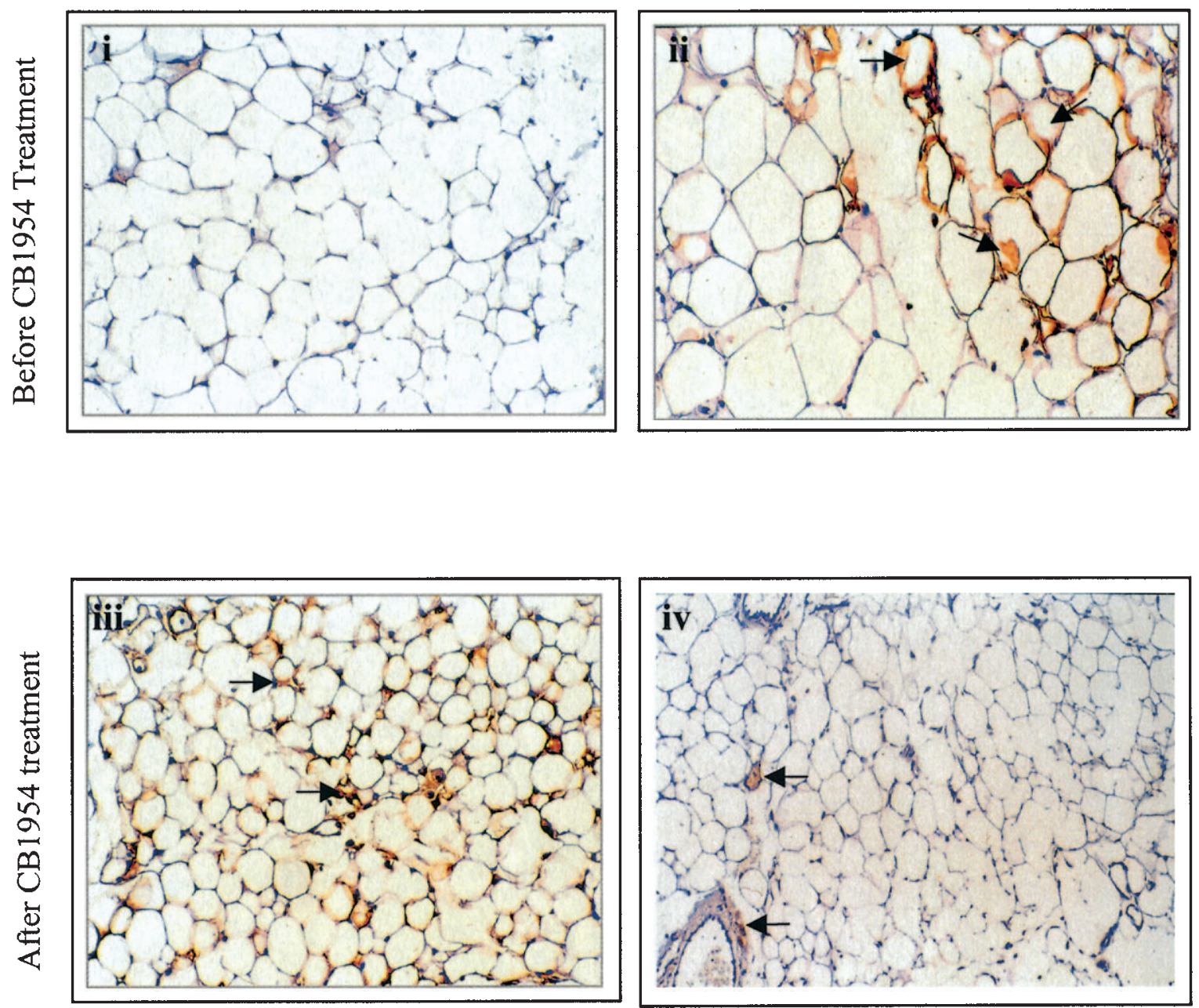

Figure 7 Immunostaining of adipose tissue. Sections of adipose tissue of a control non-transgenic mouse (i) and a transgenic mouse (ii) showing patchy staining of NTR (arrows). Panels iii and iv show sections of adipose tissue from transgenic mice treated with five injections of $20 \mathrm{mg} / \mathrm{kg}$ body weight and killed on day 5 (iii) and treated similarly and killed on day 30 (iv). Immnunostaining is observed almost exclusively between cells (arrows in panel iii) but only non-specific staining is observed in some blood vessels when killed after 30 days (arrows in panel iv). Paraffin-embedded sections were hybridised with antiserum to NTR according to the procedure described in Materials and Methods. Original Magnification: $\times 200 \mathrm{i}$ and ii, $\times 100 \mathrm{iii}$ and iv.

expressing cells (data not shown). Variegated transgene expression has been observed in mice carrying different transgenes (Robertson et al. 1996, Festenstein et al. 1996). We also have previously observed variegated expression of an NTR transgene in the mammary gland (Clark et al. 1997), and studies carried out in our laboratory have shown that this effect seems to be a consequence of the chromosome integration site of the transgene as well as the transgene copy number (Dobie et al. 1996).

This reduction of adipose tissue in transgenic mice had important implications especially in the ability to thermoregulate body temperature. Some morbidity effects could be observed after $72 \mathrm{~h}$ of treatment with some mice showing signs of cold and inactivity. More severe cases included the death of some mice. Although we could not see a direct toxic effect of the prodrug (necrosis) we cannot rule out the possibility of a toxic metabolite of the prodrug acting in a tissue other than the fat. Measurement of body temperature confirmed a marked reduction in the transgenic group. A drop in body temperature has also been previously observed in mice devoid of fat and it has been suggested that this effect is due to the effects of the loss of fat needed for heat production (Moitra et al. 1998). This group also described mortality of up to $60 \%$ by weaning in these mice. BAT is responsible for non-shivering thermogenesis, in which fuel oxidation is uncoupled from ATP synthesis to generate heat (Himms-Hagen 1990). Since the $\mathrm{aP} 2$ promoter/enhancer is expressed in both WAT and 
CB1954 5 Days

Sacrificed at day 5
CB1954 5 Days

Sacrificed at day 30

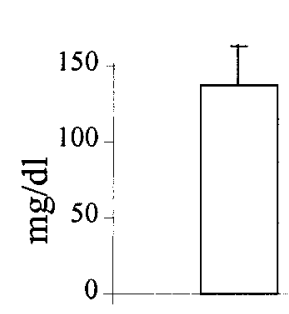

Glucose

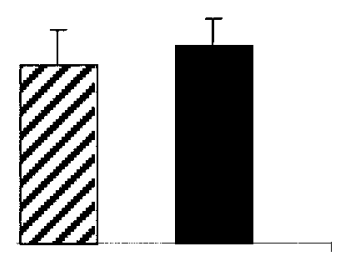

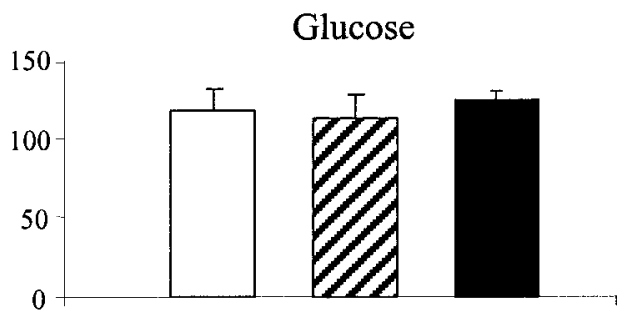

Triglycerides

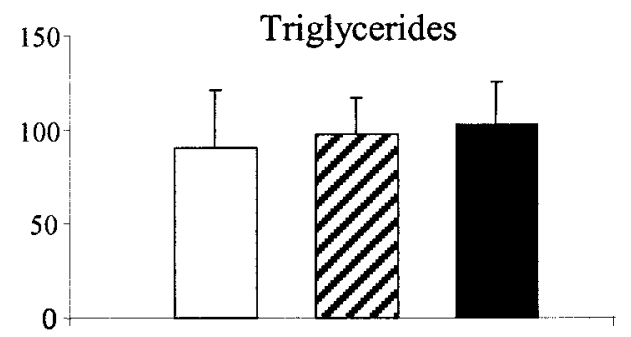

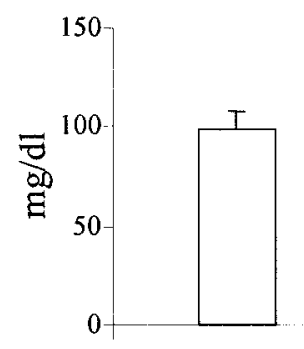

Triglycerides
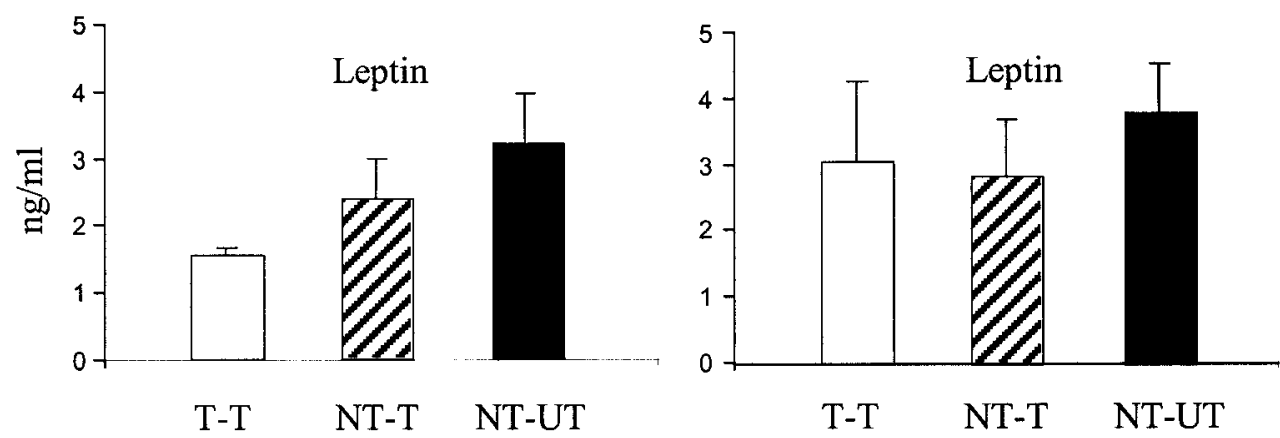

Figure 8 Physiological analyses after treatment with CB1954. Male mice (transgenic and non-transgenic) were injected with CB1954 at doses of $20 \mathrm{mg} / \mathrm{kg}$ body weight for 5 days and killed on day 5 (first group) and day 30 (second group). Significant differences were observed only in the leptin levels when the transgenic group is compared with the non-transgenic untreated group killed on day 5, however, no such difference was observed at day 30. Blood glucose level represent individual determinations ( $n=6$ each group), except in the second group killed on day 30 ( $n=5$ each group). Blood leptin and tryglicerides represent three pooled samples $(n=6)$, except in the second group killed on day 30 that represent two pooled samples. T-T: transgenic treated; NT-T: non-transgenic treated; NT-UT: non-transgenic untreated.

BAT (Ross et al. 1990) we anticipated that BAT would have been ablated. Nevertheless, analysis of the wet weight of this tissue showed only a slight non-significant reduction and the histological analysis was inconclusive in showing that non-shivering thermogenesis may have been impaired. Further experiments are required to assess the thermogenic capacity of this tissue in transgenic mice treated with the prodrug. These results are, however, consistent with reports describing two other transgenic mouse models devoid of fat, which also showed resistance to the ablation of BAT (Shimomura et al. 1998, Moitra et al. 1998). These two teams genetically blocked the growth of fat cells by altering transcription factors crucial to cell growth and differentiation and used the same aP2 promoter to direct the transgene to adipose tissue. In both cases mice were born with little or no WAT, whereas the BAT was only reduced in size in one case and enlarged with the appearance of inactive brown cells in the other. The failure to remove brown fat may correspond to an homeostatic response of the brown adipocytes to an altered metabolic status of these mice (Moitra et al. 1998) or to a less efficient killing of the cells by the prodrug.

The effects of CB1954 were not restricted to a particular adipose depot, since all major depots analysed showed 
reduction or disappearance of adipocytes. Histological analysis of sections of the skin also confirmed the removal of adipocytes in this tissue. In some mice we observed a complete disappearance of adipose depots. This suggests that a bystander effect may also occur in vivo, since immunohistochemical analysis of these mice showed a variegated pattern of expression of the transgene. In this bystander effect, a toxic metabolite of CB1954 would freely cross the cellular membrane and kill non-expressing neighbouring cells. This contrasts to studies on the ablation of mammary epithelial cells where we did not detect bystander effects (Clark et al. 1997).

In these studies we show that the adipose tissue is capable of regeneration after a prodrug free period of 30 days, although the effect could only be assessed in mice receiving the lower dose of CB1954. This supports earlier experiments carried out by Faust et al. (1977) which showed that rats that have had some portions of the epididymal fat pads removed could regenerate the missing portions by tissue hypertrophy of the remaining cell population. In other experiments, where the subcutaneous inguinal fat pad was more completely removed, regrowth of adipose tissue involved regeneration, with some compensatory hyperplasia of neighbouring fat cells (Roth et al. 1981). These two experiments clearly show that adipose tissue can regenerate but the mechanism involved may depend on the extent of the original ablation. In the present experiments, immunohistochemical analysis showed that NTR-expressing adipocytes were absent in adipose tissue from mice killed 30 days after CB1954 administration, despite the regeneration of this tissue. This might suggest that regeneration of the adipose tissue only involves the hyperplasia/hypertrophy of the remaining cell population. This is because expansion of a population of pre-adipocytes/stromal stem cells, which do not express the aP2 promoter (Ross et al. 1990), followed by differentiation and activation of the transgene in some lineages, would be expected to lead to the regeneration of some NTR expressing cells. Nevertheless, it remains to be determined how this tissue will respond to a more complete destruction of the adipocytes after CB1954 treatment.

No major physiological consequences were observed from the ablation of adipose cells with a lower dose of CB1954. Blood glucose and plasma triglyceride levels remained normal or at levels comparable to the control groups after a short ablation period of 5 days. After a prodrug free period of 30 days, blood glucose levels and triglycerides were comparable to controls thus indicating no longer term effects for these parameters. By contrast, leptin levels decreased significantly when compared with the untreated group but the levels returned to normal after 30 days, corroborating the proposed role of this hormone as an adiposity indicator. No such difference was observed when the non-transgenic treated group was compared with the untreated control group.
The results presented here clearly show the feasibility of conditionally ablating adipose cells in transgenic mice. No side effects associated with carrying or expressing the NTR transgene were observed in any of the transgenic lines generated and both sexes were fully fertile. We have recently shown that CB1954 crosses the blood-brain barrier and can be used to selectively ablate neurons (Isles et al. 2001) or glial cells (Cui et al. 2001) and preliminary experiments also show that the system can be used to ablate cells in utero (R Felmer, unpublished results) thus allowing the removal of specific cell types, providing there is specificity of the regulatory elements used to direct NTR expression to these cells, in the developing embryo. An important extension of this work would be in the in vivo ablation of adipocytes in genetically obese mice before the emergence of the morbidity signs of obesity, thus allowing the study of the interrelationship between excess of adipose tissue and the associated disorders.

\section{Acknowledgements}

We would like to thank Roberta Wallace for generating the transgenic mice and Richard Meehan for helpful discussions. The financial support of INIA, Chile (National Institute of Agriculture Research), and the Biotechnology and Biological Sciences Research Council UK, are gratefully acknowledged.

\section{References}

Ailaud G \& Hauner H 1998 Development of white adipose tissue. In Handbook of Obesity, pp 359-378. Eds GA Bray, C Bouchard \& WPT James. New York: Marcel Dekker.

Borrelli E, Heyman RA, Arias C, Sawchenko PE \& Evans RM 1989 Transgenic mice with inducible dwarfism. Nature 15 538-541.

Breitman ML, Clapoff S, Rossant J, Tsui LC, Glode LM, Maxwell IH \& Bernstein A 1987 Genetic ablation: targeted expression of a toxin gene causes microphthalmia in transgenic mice. Science $\mathbf{2 3 8}$ 1563-1565.

Burant CF, Sreenan S, Hirano K, Tai TA, Lohmiller J, Lukens J, Davidson NO, Ross S \& Graves RA 1997 Troglitazone action is independent of adipose tissue. Journal of Clinical Investigation 11 2900-2908.

Clark AJ, Iwobi M, Cui W, Crompton M, Harold G, Hobbs S, Kamalati T, Knox R, Neil C, Yull F \& Gusterson B 1997 Selective cell ablation in transgenic mice expression $E$ coli nitroreductase. Gene Therapy 2 101-110.

Cui W, Gusterson B \& Clark AJ 1999 Nitroreductase mediated cell ablation is very rapid and mediated by a p-53 independent apoptotic pathway. Gene Therapy 6 764-770.

Cui W, Allen ND, Skynner M, Gusterson B \& Clark AJ 2001 Inducible ablation of astrocytes shows that these cells are required for neuronal survival in the adult brain. Glia 34 272-282.

Dobie KW, Lee M, Fantes JA, Graham E, Clark AJ, Springbett A, Lathe R \& McClenaghan M 1996 Variegated transgene expression in mouse mammary gland is determined by the transgene integration locus. PNAS 13 6659-6664.

Drabek D, Guy J, Craig R \& Grosveld F 1997 The expression of bacterial nitroreductase in transgenic mice results in specific cell killing by the prodrug CB1954. Gene Therapy 4 93-100. 
Faust IM, Johnson PR \& Hirsch J 1977 Adipose tissue regeneration following lipectomy. Science 197 391-393.

Festenstein R, Tolaini M, Corbella P, Mamalaki C, Parrington J, Fox M, Miliou A, Jones M \& Kioussis D 1996 Locus control region function and heterochromatin-induced position effect variegation. Science 271 1123-1125.

Flier JS 1997 Leptin expression and action: new experimental paradigms. PNAS 9 4242-4245.

Himms-Hagen J 1990 Brown adipose tissue thermogenesis: interdisciplinary studies. FASEB Journal 11 2890-2898.

Houstek J, Kopecky J, Rychter Z \& Soukup T 1988 Uncoupling protein in embryonic brown adipose tissue - existence of nonthermogenic and thermogenic mitochondria. Biochimica et Biophysica Acta 1 19-25.

Isles AR, Ma D, Milsom C, Skynner MJ, Cui W, Clark AJ, Keverne EB \& Allen ND 2001 Conditional ablation of neurones in transgenic mice. Journal of Neurobiology 3 183-193.

Knox RJ, Friedlos F \& Boland MP 1993 The bioactivation of CB 1954 and its use as a prodrug in antibody-directed enzyme prodrug therapy (ADEPT). Cancer and Metastasis Reviews $\mathbf{2}$ 195-212.

Lowell BB, S-Susulic V, Hamann A, Lawitts JA, Himms-Hagen J, Boyer BB, Kozak LP \& Flier JS 1993 Development of obesity in transgenic mice after genetic ablation of brown adipose tissue. Nature 6457 740-742.

Moitra J, Mason MM, Olive M, Krylov D, Gavrilova O, Marcus-Samuels B, Feigenbaum L, Lee E, Aoyama T, Eckhaus M,
Reitman ML \& Vinson C 1998 Life without white fat: a transgenic mouse. Genes and Development 20 3168-3181.

Robertson G, Garrick D, Wilson M, Martin DI \& Whitelaw E 1996 Age-dependent silencing of globin transgenes in the mouse. Nucleic Acids Research 8 1465-1471.

Rogers P \& Webb GP 1980 Estimation of body fat in normal and obese mice. British Journal of Nutrition 43 83-86.

Ross SR, Graves RA, Greenstein A, Platt KA, Shyu HL, Mellovitz B \& Spiegelman BM 1990 A fat-specific enhancer is the primary determinant of gene expression for adipocyte $\mathrm{P} 2$ in vivo. PNAS 87 9590-9594.

Ross SR, Graves RA \& Spiegelman BM 1993 Targeted expression of a toxin gene to adipose tissue: transgenic mice resistant to obesity. Genes and Development 7 1318-1324.

Roth J, Greenwood MR \& Johnson PR 1981 The regenerating fascial sheath in lipectomized Osborne-Mendel rats: morphological and biochemical indices of adipocyte differentiation and proliferation. International Journal of Obesity 2 131-143.

Shimomura I, Hammer RE, Richardson JA, Ikemoto S, Bashmakov Y, Goldstein JL \& Brown MS 1998 Insulin resistance and diabetes mellitus in transgenic mice expressing nuclear SREBP-1c in adipose tissue: model for congenital generalized lipodystrophy. Genes and Development 20 3182-3194.

Received in final form 22 July 2002

Accepted 24 July 2002 